\title{
SOR-like methods for solving the Sylvester equation
}

\author{
Jakub Kierzkowski
}

Faculty of Mathematics and Information Science

Warsaw University of Technology

J.Kierzkowski@mini.pw.edu.pl

\section{Abstract}

We present the SOR-like methods and highlight some of their known properties. We give the SOR-like method as proposed by Z. Woźnicki and propose two similar methods based upon it. All three are stationary iterative methods for solving the Sylvester equation $(A X-X B=C)$. We form two sufficient conditions under which one of those methods will converge. In addition, we present a modification method, based on the following fact: if $X$ is a solution of $A X-X B=C$, it is also a solution of $\left(A-\alpha I_{m}\right) X-X\left(B-\alpha I_{n}\right)=C$.

We also present numerical experiments to illustrate the theoretical results and some properties of the methods.

\section{Introduction}

Definition 1. Let $A \in \mathbb{R}^{m \times m}, B \in \mathbb{R}^{n \times n}, C \in \mathbb{R}^{m \times n}$ be given matrices, and $X \in \mathbb{R}^{m \times n}$ be an unknown matrix. The Sylvester equation then is the equation of the form

$$
A X-X B=C \text {. }
$$

The Sylvester equation has many applications, for example in control theory and when solving partial differential equations numerically.

Let $A$ be cut into parts $A=D-L-U$, where $L$ and $U$ are lower and upper triangular, with zeros on the main diagonals, respectively, and $D$ is nonsingular diagonal:

$$
\begin{gathered}
\text { Algorithm 1: SOR-like method } \\
X^{(t)}=\left(I-\omega D^{-1} L\right)^{-1}\left(\left((1-\omega) I+\omega D^{-1} U\right) X^{(t-1)}+\omega D^{-1}\left(X^{(t-1, t)} B+C\right)\right)
\end{gathered}
$$

The most characteristic idea in this method is the usage of the double index $(t-1, t)$ - we take the entries of the latest approximation $X^{(t)}$ if they are already computed in the $t$-th iteration, and entries of the previous approximation $X^{(t-1)}$ for all the rest of the entries.

To avoid such difficulties, we can modify the method's formula by replacing the double index $(t-1, t)$ with the simple $(t-1)$ :

$$
\begin{gathered}
\text { Algorithm 2: ISOR-like method } \\
X^{(t)}=\left(I-\omega D^{-1} L\right)^{-1}\left(\left((1-\omega) I+\omega D^{-1} U\right) X^{(t-1)}+\omega D^{-1}\left(X^{(t-1)} B+C\right)\right)
\end{gathered}
$$

On the other hand, if there already is the double index in the formula, it can be used in all occurrences of the matrix $X$, hoping that it will improve the convergence:

$$
\begin{gathered}
\text { Algorithm 3: bSOR-like method } \\
X^{(t)}=\left(I-\omega D^{-1} L\right)^{-1}\left(\left((1-\omega) I+\omega D^{-1} U\right) X^{(t-1, t)}+\omega D^{-1}\left(X^{(t-1, t)} B+C\right)\right)
\end{gathered}
$$

If $X$ is a solution of $A X-X B=C$, it is also a solution of $\left(A-\alpha I_{m}\right) X-X(B-$ $\left.\alpha I_{n}\right)$ for all $\alpha$. Any of the SOR-like methods can be used to solve the modified problem with $\alpha \neq 0$. This can be also treated in a different way, as using a different (more general) method for the basic problem $A X-X B=C$, where $\alpha$ is a method's parameter, like $\omega$ is. We call this approach the $\alpha$ SOR-like methods. Any SORlike method is a special case of its corresponding $\alpha$ SOR-like method for $\alpha=0$.

$$
\begin{gathered}
\text { Algorithm 4: } \alpha \text { ISOR-like method } \\
X^{(t)}=\left(I-\omega\left(D-\alpha I_{m}\right)^{-1} L\right)^{-1} \cdot \\
\cdot\left(\left((1-\omega) I+\omega\left(D-\alpha I_{m}\right)^{-1} U\right) X^{(t-1)}+\omega D^{-1}\left(X^{(t-1)} B-\alpha X^{(t-1)}+C\right)\right)
\end{gathered}
$$

$\alpha$ SOR-like and $\alpha$ bSOR-like methods are defined the same way.

\section{ISOR-like convergence}

Let $A \in \mathbb{R}^{m \times m}, B \in \mathbb{R}^{n \times n}, A \neq 0, B \neq 0$ and $\operatorname{det}(D) \neq 0$.

Theorem 1 (First sufficient condition for ISOR-like convergence)

Let $\mathbf{G}=\left\|D^{-1} L\right\|\left(1+\left\|D^{-1} U\right\|+\left\|D^{-1}\right\| \cdot\|B\|\right), \mathbf{H}=1+\left\|D^{-1} U\right\|+\left\|D^{-1}\right\| \cdot\|B\|-\left\|D^{-1} L\right\|$,

$\mathbf{M}=\left\|D^{-1} L\right\|\left(-1+\left\|D^{-1} U\right\|+\left\|D^{-1}\right\| \cdot\|B\|\right), \mathbf{N}=-1+\left\|D^{-1} U\right\|+\left\|D^{-1}\right\| \cdot\|B\|+\left\|D^{-1} L\right\|$.

Then if $\left\|I-\omega D^{-1} L\right\| \geq\left\|\left(I-\omega D^{-1} L\right)^{-1}\right\|$ and

$$
\left\{\begin{array}{l}
\mathbf{G} \cdot \omega^{2}+\mathbf{H} \cdot \omega-2<0 \text { for } \omega \geq 1 \\
\mathbf{M} \cdot \omega^{2}+\mathbf{N} \cdot \omega<0 \text { for } 0<\omega \leq 1
\end{array}\right.
$$

then ISOR-like converges.

Note that for $\omega=1, \mathbf{G} \cdot \omega^{2}+\mathbf{H} \cdot \omega-2=\mathbf{M} \cdot \omega^{2}+\mathbf{N} \cdot \omega$

Theorem 2 (Second sufficient condition for ISOR-like convergence).

If $\omega>0$ and

$$
\frac{|1-\omega|+\omega\left\|D^{-1} U\right\|+\omega\left\|D^{-1}\right\|\|B\|}{1-\omega\left\|D^{-1} L\right\|} \in(0 ; 1)
$$

then ISOR-like converges

\section{Numerical tests}

3.1 Example 1. Numerical solution for Laplace's PDE

$-\Delta u=2 \sin (y)(x \sin (x)-\cos (x)), u_{\mid \delta U}=0, U=[0, \pi] \times[0,2 \pi], A \in \mathbb{R}^{12 \times 12}, B \in \mathbb{R}^{25 \times 25}$,

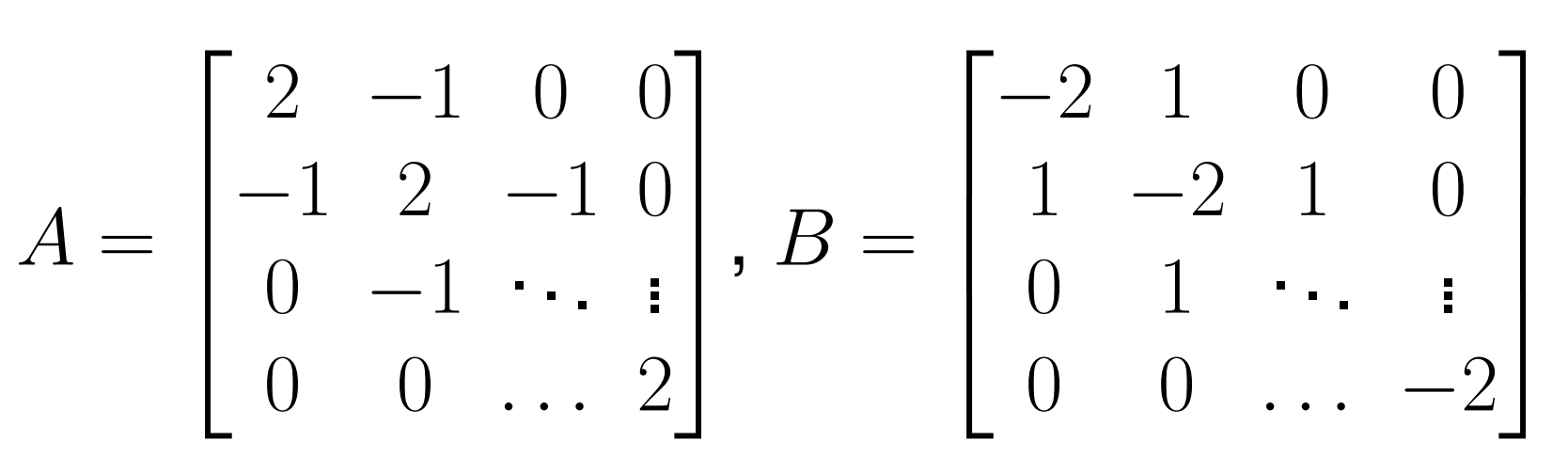

$\alpha=[-11,-10.5,-10, \ldots, 1.5,2,2.5], \quad \omega=[0.1,0.2,0.3, \ldots, 4.8,4.9,5], C \in \mathbb{R}^{12 \times 25}$ is from the right hand side function, initial guess $X^{(0)}=C$.

Table 1: $\alpha$ and $\omega$ values for which the methods converged the fastest

method $\alpha_{\text {opt }} \omega_{\text {opt }}$ iterations

alSOR-like $-6.5 \quad 2.8 \quad 750^{*}$

$\alpha$ SOR-like $\quad-2 \quad 1.7 \quad 89$

abSOR-like $\quad-5 \quad 2.9 \quad 311$

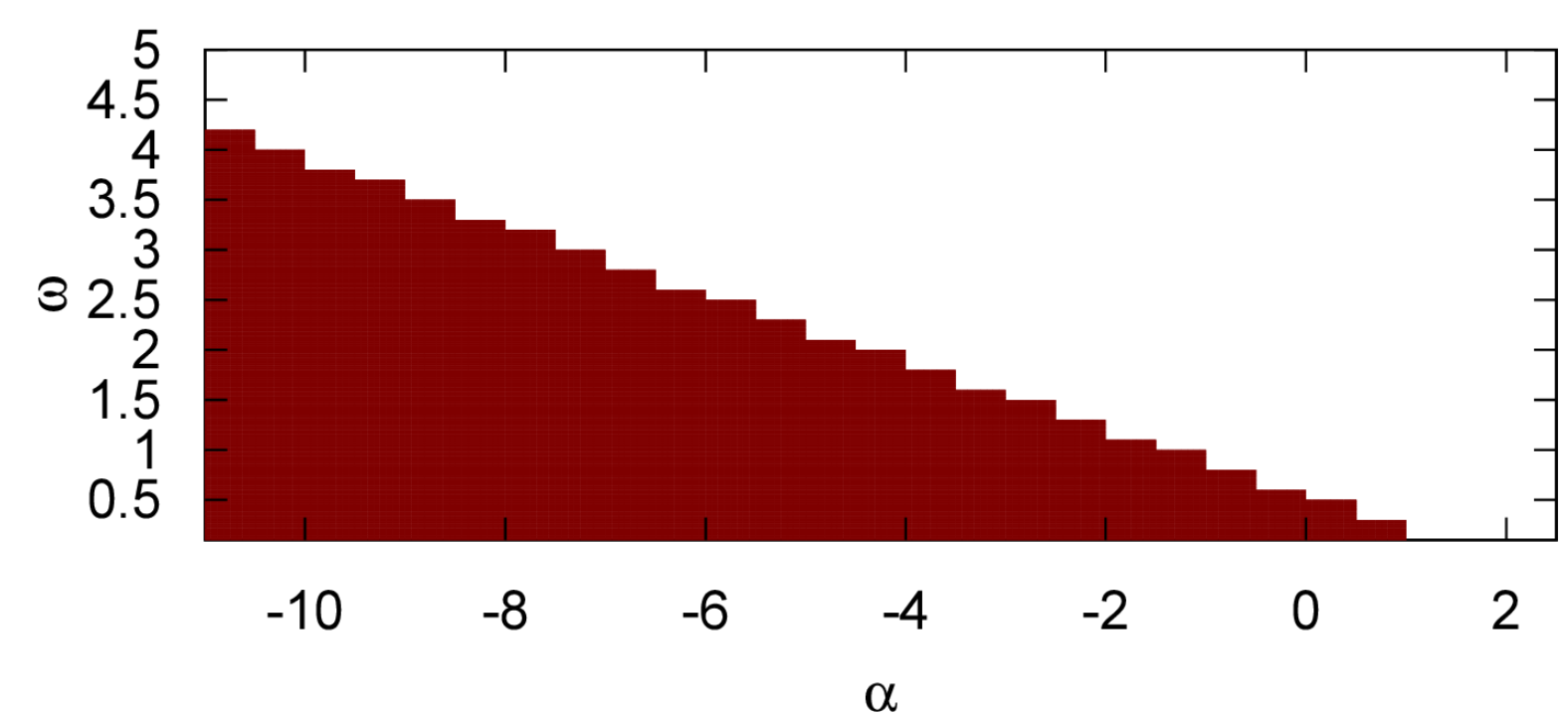
verged

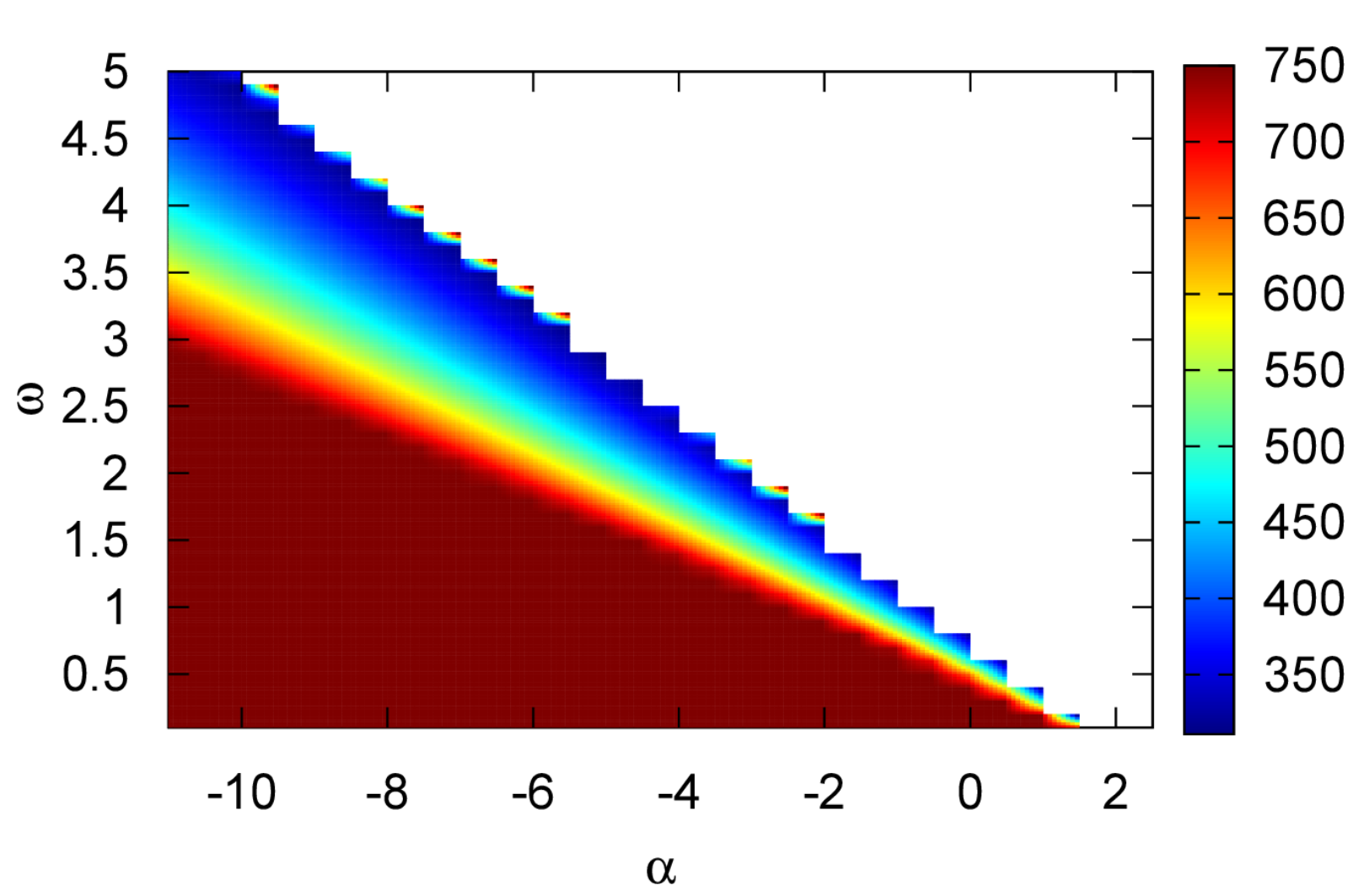

(a) bSOR-like

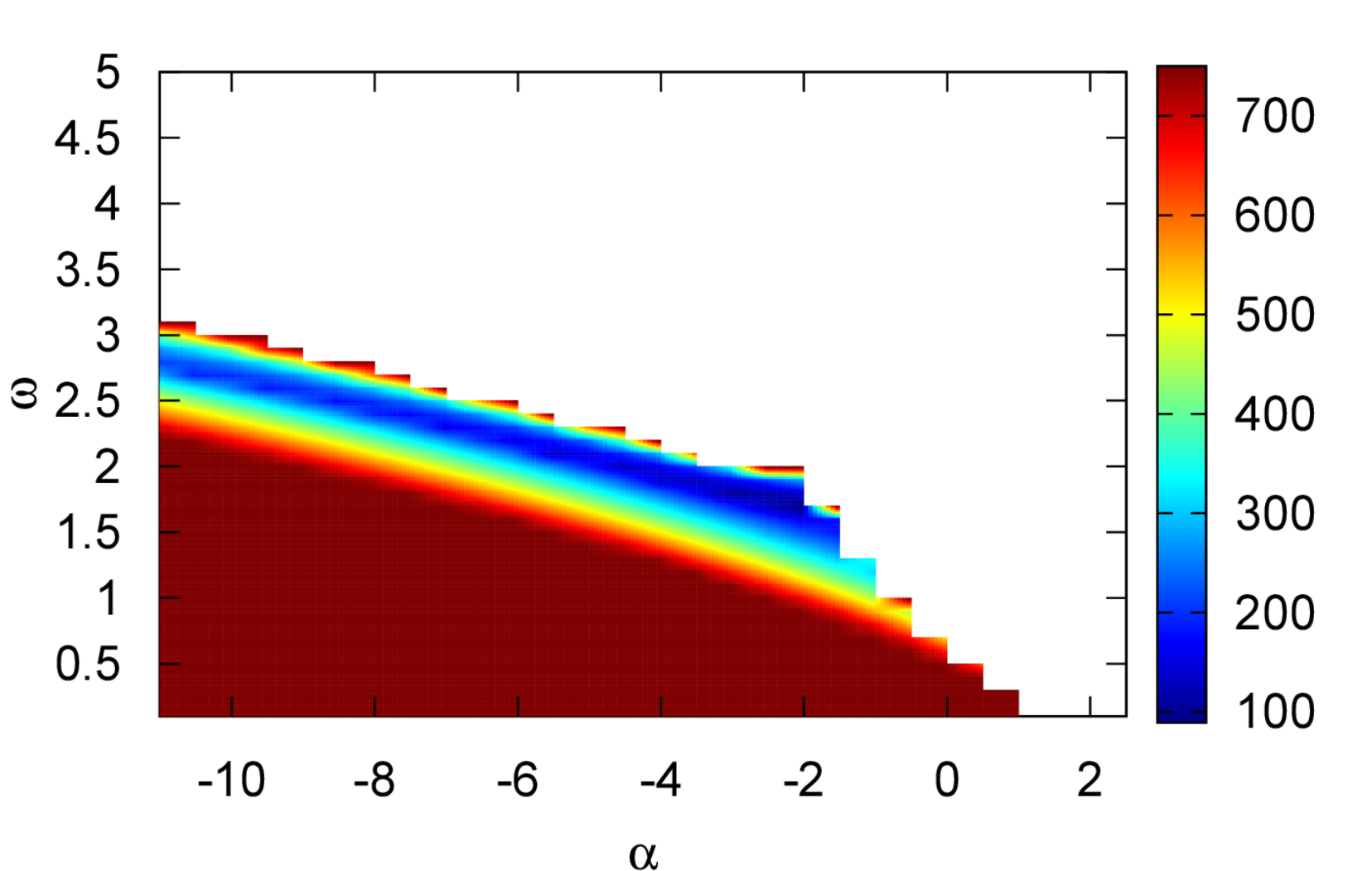

(b) SOR-like
Figure 2: Numbers of iterations for $(\alpha, \omega)$ pairs

The conditions of Theorem 1 were not satisfied for any of the tested values. Among the tested values, the conditions of Theorem 2 were satisfied for $\alpha=[-11,-10.5, \ldots,-2]$ and $\omega=[0.1,0.2, \ldots, 1]$. All the SOR-like methods did converge for that values.

\subsection{Example 2.}

For given $\alpha$ and $\omega$, using the Kronecker product and the vectorization operation (see eg. [1]), Algorithm 4. can be written in the form $\vec{X}^{(t)}=M \vec{X}^{(t-1)}+\vec{Z}$, where $M$ is a transition matrix. In this example we present the value of the spectral radius of $M$ depending on $\alpha$ and $\omega$.

$$
A=\left[\begin{array}{cccc}
10 & 1 & \cdots & 1 \\
1 & 11 & \cdots & 1 \\
\vdots & \vdots & \cdots & \vdots \\
1 & 1 & \cdots & 19
\end{array}\right], B=\left[\begin{array}{ll}
0.382 & 0.382 \\
0.382 & 0.764
\end{array}\right]
$$

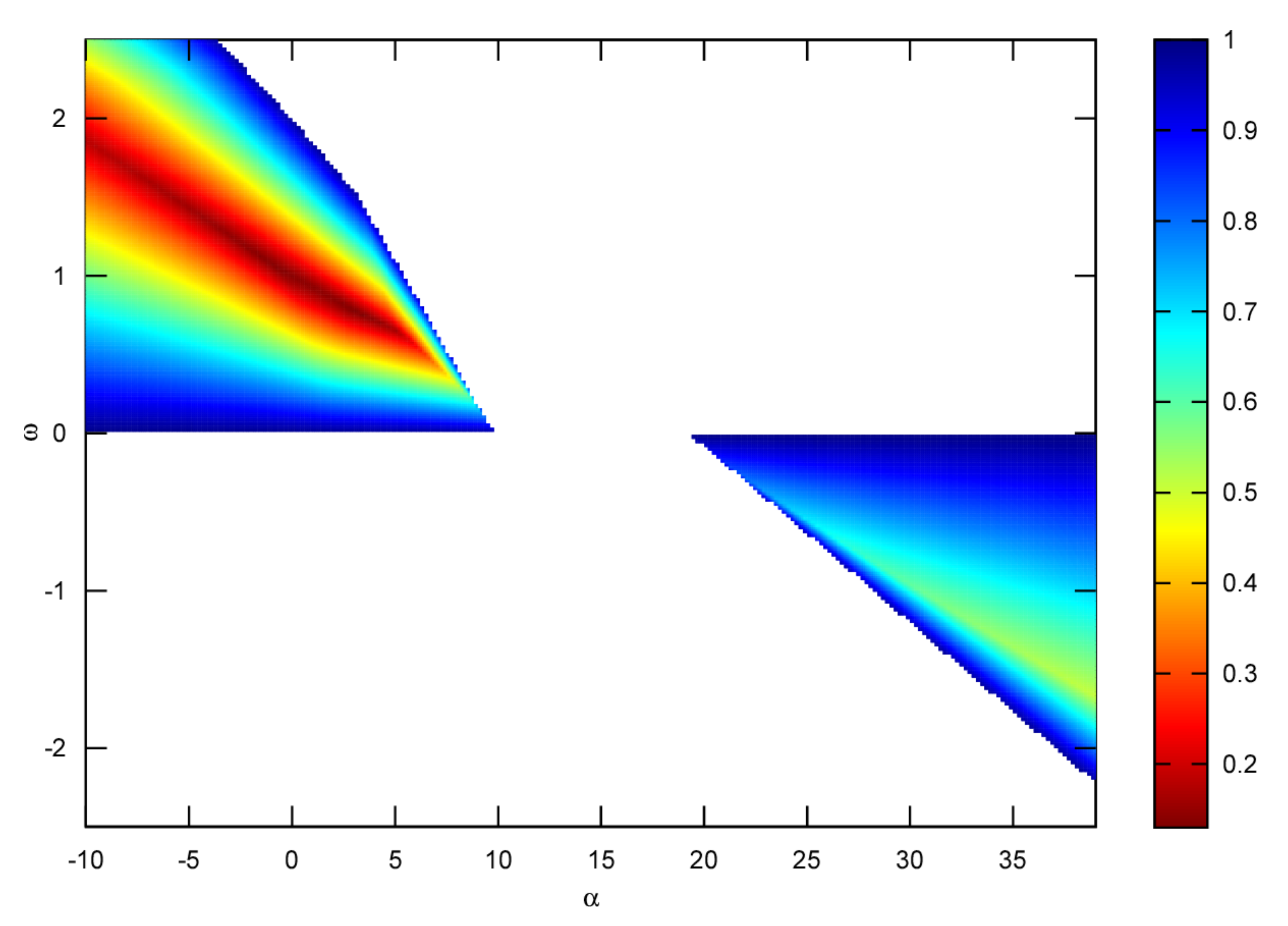

Figure 3: Spectral radius of $M$. White color for values greater than 1.

The $\alpha$ ISOR method does not converge for $\alpha \in(10,19)$, where 10 and 19 are the minimum and maximum of the diagonal of $A$. More, for big enough $\alpha$, the method converge for $\omega<0$.

\section{References}

[1] J.Kierzkowski, New SOR-like methods for solving the Sylvester equation. Open Mathematics 13(1), pages 178-187, 2015.

[2] Z. Woźnicki, Solving Linear Systems: An Analysis of Matrix Prefactorization Iterative Methods. Matrix Editions, 2009. 